\title{
Star Formation and Gas Dynamics in Galactic Disks: Physical Processes and Numerical Models
}

\author{
Eve C. Ostriker \\ Department of Astronomy, University of Maryland, College Park, MD 20742; \\ email: ostriker@astro.umd.edu
}

\begin{abstract}
Star formation depends on the available gaseous "fuel" as well as galactic environment, with higher specific star formation rates where gas is predominantly molecular and where stellar (and dark matter) densities are higher. The partition of gas into different thermal components must itself depend on the star formation rate, since a steady state distribution requires a balance between heating (largely from stellar UV for the atomic component) and cooling. In this presentation, I discuss a simple thermal and dynamical equilibrium model for the star formation rate in disk galaxies, where the basic inputs are the total surface density of gas and the volume density of stars and dark matter, averaged over $\sim$ kpc scales. Galactic environment is important because the vertical gravity of the stars and dark matter compress gas toward the midplane, helping to establish the pressure, and hence the cooling rate. In equilibrium, the star formation rate must evolve until the gas heating rate is high enough to balance this cooling rate and maintain the pressure imposed by the local gravitational field. In addition to discussing the formulation of this equilibrium model, I review the current status of numerical simulations of multiphase disks, focusing on measurements of quantities that characterize the mean properties of the diffuse ISM. Based on simulations, turbulence levels in the diffuse ISM appear relatively insensitive to local disk conditions and energetic driving rates, consistent with observations. It remains to be determined, both from observations and simulations, how mass exchange processes control the ratio of cold-to-warm gas in the atomic ISM.
\end{abstract}

\section{Introduction}

Disk galaxies are gas-rich systems, with a multi-phase, highly structured interstellar medium (ISM). Within the ISM, star formation takes place in giant molecular clouds (GMCs), sometimes concentrated in spiral arms. The rate and character of star formation are influenced by physical processes from sub-pc to multi-kpc scales (McKee \& Ostriker 2007). In spite of the complexity of the ISM and star formation at small scales, there are nevertheless clear correlations between the large-scale rate at which stars are born, and the properties of the ISM and (intra-)galactic environment on large $(\sim \mathrm{kpc})$ scales.

As discussed by Frank Bigiel at this meeting (see also Bigiel et al. 2008, and references therein), in regions of galaxies where the gaseous surface density $\Sigma \lesssim 100 \mathrm{M}_{\odot} \mathrm{pc}^{-2}$, the star formation rate closely follows the surface density of molecular gas. This can be understood in terms of the gas having an essentially constant star formation timescale, $t_{\mathrm{SF}} \sim 2 \times 10^{9} \mathrm{yr}$, within molecular gas (which is observed to be in organized in gravitationally bound clouds with properties that are similar in different galaxies). As a consequence, $\Sigma_{\mathrm{SFR}} \propto \Sigma$ in regions where the molecular gas dominates the atomic gas. For regions where atomic gas dominates (primarily in the outer parts of galaxies), $\Sigma_{\mathrm{SFR}}$ instead varies as a steeper power of $\Sigma$. In addition to this superlinear behavior, there is considerable scatter in the relation between $\Sigma_{\text {SFR }}$ vs. $\Sigma$ at low surface density, suggesting that one or more other parameters, in addition to $\Sigma$, controls the star formation rate. 
Indeed, recent examination of the correlation of $\Sigma_{\text {SFR }}$ with "non-interstellar" galactic environmental properties has revealed interesting dependences, indicating that in the outer parts of galaxies, both the specific star formation rate and the ratio of molecularto-atomic gas increase roughly linearly with the stellar surface density $\Sigma_{s}$ (Leroy et al. 2008). Previously, Blitz \& Rosolowsky (2006) found an approximately linear increase of the molecular content with the estimated dynamic pressure of the ISM, and this is evident in the sample analyzed by Leroy et al. (2008) as well. The physical reason for the relationship between molecular content (and star formation) and pressure has not, however, been clear from these empirical studies.

Observations of star formation pose a number of challenges: Why is there an increase in the slope of $\Sigma_{\mathrm{SFR}} \propto \Sigma^{1+p}$ in going from molecular- to atomic-dominated regions? What is the physical reason for the empirical relation between ISM pressure and star formation; more generally, how do galactic parameters such as $\Sigma_{s}$, the velocity dispersions of stars and of gas, and spiral structure affect $\Sigma_{\mathrm{SFR}}$ ? Is it possible to explain the observed behavior of $\Sigma_{\text {SFR }}$ using simplified theoretical models, and what is required in numerical simulations in order to reproduce observed star formation relationships? Recent theoretical work has taken on these challenges with increasing success; a key to these advances has been a more sophisticated treatment of both the ISM and the galactic environment. For example, Koyama \& Ostriker (2009a) found, using numerical simulations of the ISM and a cooling function allowing multiple phases, star formation rates and proportions between selfgravitating and diffuse gas similar to the observations of Blitz \& Rosolowsky (2006) and Leroy et al. (2008) provided that turbulent driving is included; for non-turbulent models, the proportion of self-gravitating gas was found to be much too high.

\section{A thermal/dynamical equilibrium model for $\Sigma_{\mathrm{SFR}}$}

Motivated by recent observations as well as simulations and earlier theory, Ostriker et al. (2010) (hereafter OML) have developed a simple model for star formation regulation in multiphase, turbulent ISM disks. In essence, the OML model combines three basic principles: (1) the diffuse (atomic) component of the ISM is in approximate thermal equilibrium, with a density (and pressure) proportional to the heating rate; (2) the diffuse component of the ISM is in approximate dynamical equilibrium, with the pressure at any height above the galactic midplane given by the weight of the overlying gas; (3) UV from young stars provides most of the heating for the atomic component of the ISM, with star formation taking place only within the gravitationally-bound component of the ISM. These principles have been individually established and extensively studied (over several decades) in the astrophysical literature. Field et al. (1969) combined (1) and (2) to conclude that the diffuse atomic gas in the local Milky Way must consist of a two-phase cloud/intercloud medium. In this and subsequent treatments of thermal and dynamical equilibrium, the heating rate has generally been treated as an independent (empirical) parameter. But, by including (3) together with (1) and (2), OML obtained a closed system representing a local patch of a disk galaxy. For this closed system, the partition of gas into phases and the star formation rate are obtained self-consistently.

In the OML model, the (simplified) ISM is treated as having two components, one consisting of diffuse gas (including both high-density cold atomic cloudlets and a lowdensity warm atomic intercloud medium), and the other consisting of gravitationallybound clouds (GBCs). Although hot gas is also present in the ISM, it is a tiny fraction of the mass, and fills $\lesssim 20 \%$ of the volume (Heiles 2001) (OML describe how to correct for this effect). For galaxies with normal metallicity, the GBCs would represent giant molecular clouds, including their atomic shielding layers. Averaged over $\sim \mathrm{kpc}$ scales 
(which may contain many or few individual GBCs), the total surface density of the GBC component is $\Sigma_{\mathrm{GBC}}$, and the total surface density of the diffuse component is $\Sigma_{\text {diff }}$.

The diffuse component is assumed to be in vertical dynamical equilibrium (as has been verified by numerical simulations; e.g. Piontek \& Ostriker 2007; Koyama \& Ostriker 2009 b), with the vertical gravity (from the diffuse gas, the GBC component, the stellar disk, and the dark matter halo) balanced by the difference between midplane and external values of thermal pressure $P_{\mathrm{th}}$, turbulent pressure $\rho v_{z}^{2}$, and magnetic stresses $(8 \pi)^{-1}\left(B^{2}-\right.$ $\left.2 B_{z}^{2}\right)$. Because cooling times are short compared to other timescales, the diffuse gas is assumed to be in thermal equilibrium, with the additional provision that both warm and cold phases are present. This allows a range of pressures between $P_{\min \text {,cold }}$ and $P_{\max \text {,warm }}$; for the model of OML, it is assumed that the pressure is equal to the geometric mean of these limits, $P_{\text {two-phase }} \equiv\left(P_{\text {min,cold }} P_{\text {max,warm }}\right)^{1 / 2}$. For atomic gas, heating is generally dominated by the UV and cooling by collisionally-excited lines (Wolfire et al. 2003), which yields $P_{\text {two-phase }} \propto J_{\mathrm{UV}}$. (Note that other heating - e.g. cosmic rays and shocks - can be more important for very dense, shielded cores and very hot gas, respectively.) Finally, the OML model assumes that the rate of star formation is proportional to the total surface density $\Sigma_{\mathrm{GBC}}$ of gas in the GBC component, $\Sigma_{\mathrm{SFR}}=\Sigma_{\mathrm{GBC}} / t_{\mathrm{SF}}=\left(\Sigma-\Sigma_{\text {diff }}\right) / t_{\mathrm{SF}}$.

Vertical dynamical equilibrium within the diffuse layer is expressed as $P_{\text {tot }} \equiv \alpha P_{\text {th }}=$ $\Sigma_{\text {diff }}\left\langle g_{z}\right\rangle / 2$, where the mean vertical gravity is

$$
\left\langle g_{z}\right\rangle \approx \pi G\left(\Sigma_{\mathrm{diff}}+2 \Sigma_{\mathrm{GBC}}\right)+2\left(2 G \rho_{\mathrm{sd}}\right)^{1 / 2} \sigma_{z}
$$

$\rho_{\text {sd }}$ is the midplane density of stars plus dark matter, $\sigma_{z}$ is the total vertical velocity dispersion of the diffuse gas, and the total pressure is larger than the thermal pressure by a factor $\alpha$ (see below). The GBC component contributes more strongly (per unit mass) to the gravity because its scale height is smaller than that of the diffuse gas.

If $n^{2} \Lambda(T)$ is the cooling rate per unit volume and $n \Gamma$ is the heating rate per unit volume, then the two-phase pressure is given by

$$
\begin{aligned}
\frac{P_{\mathrm{two}-\mathrm{phase}}}{k} & \equiv\left(n_{\mathrm{min}, \text { cold }} T_{\mathrm{min}, \text { cold }} n_{\mathrm{max}, \mathrm{warm}} T_{\mathrm{max}, \mathrm{warm}}\right)^{1 / 2} \\
& =\Gamma \frac{\left(T_{\text {min }, \text { cold }} T_{\max , \mathrm{warm}}\right)^{1 / 2}}{\left[\Lambda\left(T_{\mathrm{min}, \text { cold }}\right) \Lambda\left(T_{\mathrm{max}, \mathrm{warm}}\right)\right]^{1 / 2}}
\end{aligned}
$$

where we have used the equilibrium condition $\Gamma=n \Lambda$ for both phases. Cooling of the cold atomic medium is dominated by metals (in particular, C II) so that $\Lambda \propto Z_{\text {gas }}$, while heating is dominated by the photoelectric effect with $\Gamma \propto Z_{\mathrm{dust}} J_{\mathrm{UV}}$; since $T_{\mathrm{min} \text {, cold }}$ and $T_{\max \text {,warm }}$ are relatively independent of the heating rate (Wolfire et al. 1995), this yields $P_{\text {two-phase }} \propto J_{\mathrm{UV}}$ if $Z_{\text {dust }} / Z_{\text {gas }}=$ const. The terms $Z_{\text {gas }}$ and $Z_{\text {dust }}$ represent the ratios of metals and dust to hydrogen, respectively. The mean UV intensity is affected by radiative transfer through the diffuse gas, but for modest optical depth in the diffuse gas the relation $J_{\mathrm{UV}} \propto \Sigma_{\mathrm{SFR}}$ is expected to hold. In addition, a larger fraction of the UV escapes from GBCs if $Z_{d}$ is very sub-Solar, which increases $J_{\mathrm{UV}}$ for a given $\Sigma_{\mathrm{SFR}}$ (this effect is quite uncertain, but might increase $J_{\mathrm{UV}}$ by a factor $\sim 2$ ). In thermal equilibrium with $P_{\text {th }} \sim P_{\text {two-phase }}$, the midplane pressure is therefore expected to vary roughly as $P_{\mathrm{th}} \propto \Sigma_{\mathrm{SFR}}$, with a somewhat larger coefficient for very low-metallicity regions.

Combining the thermal equilibrium relation $P_{\mathrm{th}}=P_{\mathrm{th}, 0} \Sigma_{\mathrm{SFR}} / \Sigma_{\mathrm{SFR}, 0}$ (normalized using the Solar neighborhood thermal pressure $P_{\mathrm{th}, 0}$ and star formation rate $\left.\Sigma_{\mathrm{SFR}, 0}\right)$ with the dynamical equilibrium relation $P_{\mathrm{th}}=\Sigma_{\mathrm{diff}}\left\langle g_{z}\right\rangle /(2 \alpha)$ and the star formation relation 
$\Sigma_{\mathrm{SFR}}=\Sigma_{\mathrm{GBC}} / t_{\mathrm{SF}}$, we obtain

$$
\frac{\Sigma_{\mathrm{GBC}}}{\Sigma_{\mathrm{diff}}}=\frac{\left\langle g_{z}\right\rangle}{g_{*}} \propto \pi G\left(\Sigma_{\mathrm{diff}}+2 \Sigma_{\mathrm{GBC}}\right)+2\left(2 G \rho_{\mathrm{sd}}\right)^{1 / 2} \sigma_{z} .
$$

Here, $g_{*}=2 \alpha P_{\mathrm{th}, 0} /\left(\Sigma_{\mathrm{SFR}, 0} t_{\mathrm{SF}}\right)$; for fiducial parameters, this acceleration is $g_{*} \sim \mathrm{pc} \mathrm{Myr}^{-2}$.

It is interesting to compare outer and inner disks. In outer disks (similar to the Solar neighborhood and beyond, in galaxies like the Milky-Way), diffuse gas dominates the total so that $\Sigma_{\mathrm{GBC}} \ll \Sigma_{\text {diff }} \approx \Sigma$; in addition, the term depending on $\rho_{\text {sd }}$ dominates the gravity $g_{z}$. In this regime, the relation $\Sigma_{\mathrm{SFR}} \propto \Sigma_{\mathrm{GBC}} \propto \Sigma \sqrt{\rho_{\mathrm{Sd}}}$ is therefore expected to hold. Physically, this regime may be thought of as the result of star formation increasing until the heating it provides is sufficient to balance cooling at the (dynamically-imposed) midplane pressure. If there is too little gas in the GBC component, the star formation rate would be extremely low, and the UV field would be very weak. A very low heating rate could not maintain a warm medium at the pressure imposed by the local gravitational field, so that (a portion of the) warm gas would condense out and become cold clouds. These cold clouds would collect to create more GBCs, which would then initiate star formation, raising the local UV radiation field until heating balances cooling. Given the low gravity and pressure of outer disks, cooling rates are moderate, and relatively low levels of star formation are needed to produce enough UV that heating balances cooling.

For outer disks where the stars and dark matter dominate gravity, the vertical oscillation time is $t_{\mathrm{osc}}=\pi^{1 / 2} /\left(G \rho_{\mathrm{sd}}\right)^{1 / 2}$; a dense cloud settles to the midplane in $\sim t_{\mathrm{osc}} / 4$. In this regime, the conversion time from gas to stars, $t_{\text {con }} \equiv \Sigma / \Sigma_{\mathrm{SFR}}$, is given by

$$
t_{\mathrm{con}}=t_{\mathrm{osc}} \frac{\sigma_{z} P_{\mathrm{th}, 0}}{(2 \pi)^{1 / 2}\left\langle v_{\mathrm{th}}^{2}\right\rangle \Sigma_{\mathrm{SFR}, 0}},
$$

where $\left\langle v_{\mathrm{th}}^{2}\right\rangle \equiv \tilde{f}_{w} c_{w}^{2} \approx c_{w}^{2} M_{\mathrm{diff}, \text { warm }} / M_{\mathrm{diff}, \text { total }}$ is the mean thermal dispersion in the diffuse medium (here $c_{w} \sim 8 \mathrm{~km} \mathrm{~s}^{-1}$ is the thermal speed in the warm ISM). Using $P_{\mathrm{th}, 0} \sim\left\langle v_{\mathrm{th}}^{2}\right\rangle P_{\mathrm{gas}, 0} / \sigma_{z}^{2}$ and defining a star formation energy conversion efficiency $\varepsilon_{\mathrm{rad}} \equiv$ $4 \pi J_{\mathrm{rad}, 0} /\left(c^{2} \Sigma_{\mathrm{SFR}, 0}\right)$ for $P_{\mathrm{rad}, 0}=4 \pi J_{\mathrm{rad}, 0} /(3 c)$,

$$
t_{\mathrm{con}}=t_{\mathrm{osc}} \frac{c}{3(2 \pi)^{1 / 2} \sigma_{z}} \frac{P_{\mathrm{gas}, 0}}{P_{\mathrm{rad}, 0}} \varepsilon_{\mathrm{rad}} .
$$

That is, the gas conversion time (or depletion time) is set by the time for gas to settle to the midplane, scaled by factors for the ratio of gas-to-radiation pressure in the Solar neighborhood, the mass-to-energy conversion efficiency, and $c / \sigma_{z}$.

In inner disks, unlike outer disks, we have $\Sigma_{\text {diff }} \ll \Sigma_{\mathrm{GBC}} \approx \Sigma$, so that $\Sigma_{\mathrm{SFR}} \propto \Sigma$. In inner disks, it is straightforward to show that there is an upper limit on the diffuse gas surface density $\Sigma_{\text {diff }}$. Physically, the reason for this limit is that the diffuse-gas cooling rate per particle increases with higher density and pressure in the inner parts of disks at least as $n \Lambda \propto \Sigma_{\text {diff }} \Sigma_{\mathrm{GBC}}$ (since $n \Lambda \propto \Sigma_{\text {diff }} / H \propto \Sigma_{\text {diff }} g_{z} / \sigma_{z}^{2} \propto \Sigma_{\text {diff }} \Sigma_{\mathrm{GBC}}[1+$ $\left.g_{\mathrm{sd}} / g_{\mathrm{GBC}}\right] / \sigma_{z}^{2}$ ), whereas the heating rate per particle varies as $\Gamma \propto \Sigma_{\mathrm{SFR}} \propto \Sigma_{\mathrm{GBC}}$. Thus, cooling will exceed heating (causing mass to drop out of the diffuse component) unless $\Sigma_{\text {diff }}$ is sufficiently low. Enhanced cooling and mass "dropout" is likely responsible at least in part for the "saturation" of HI surface densities at $\lesssim 10 \mathrm{M}_{\odot} \mathrm{pc}^{-2}$ that has been observed in the inner parts of galaxies (Bigiel et al. 2008).

Based on the relations described above, the star formation law is expected to steepen from $\Sigma_{\mathrm{SFR}} \propto \Sigma$ in inner disks to $\Sigma_{\mathrm{SFR}} \propto \Sigma \sqrt{\rho_{\mathrm{Sd}}}$ in outer disks. A reduction of the specific star formation rate $\Sigma_{\mathrm{SFR}} / \Sigma$ is indeed observed in galaxies starting at $\Sigma \lesssim 10 \mathrm{M}_{\odot} \mathrm{pc}^{-2}$ (Bigiel et al. 2008; Leroy et al. 2008). For some galaxies, a further power-law relation 

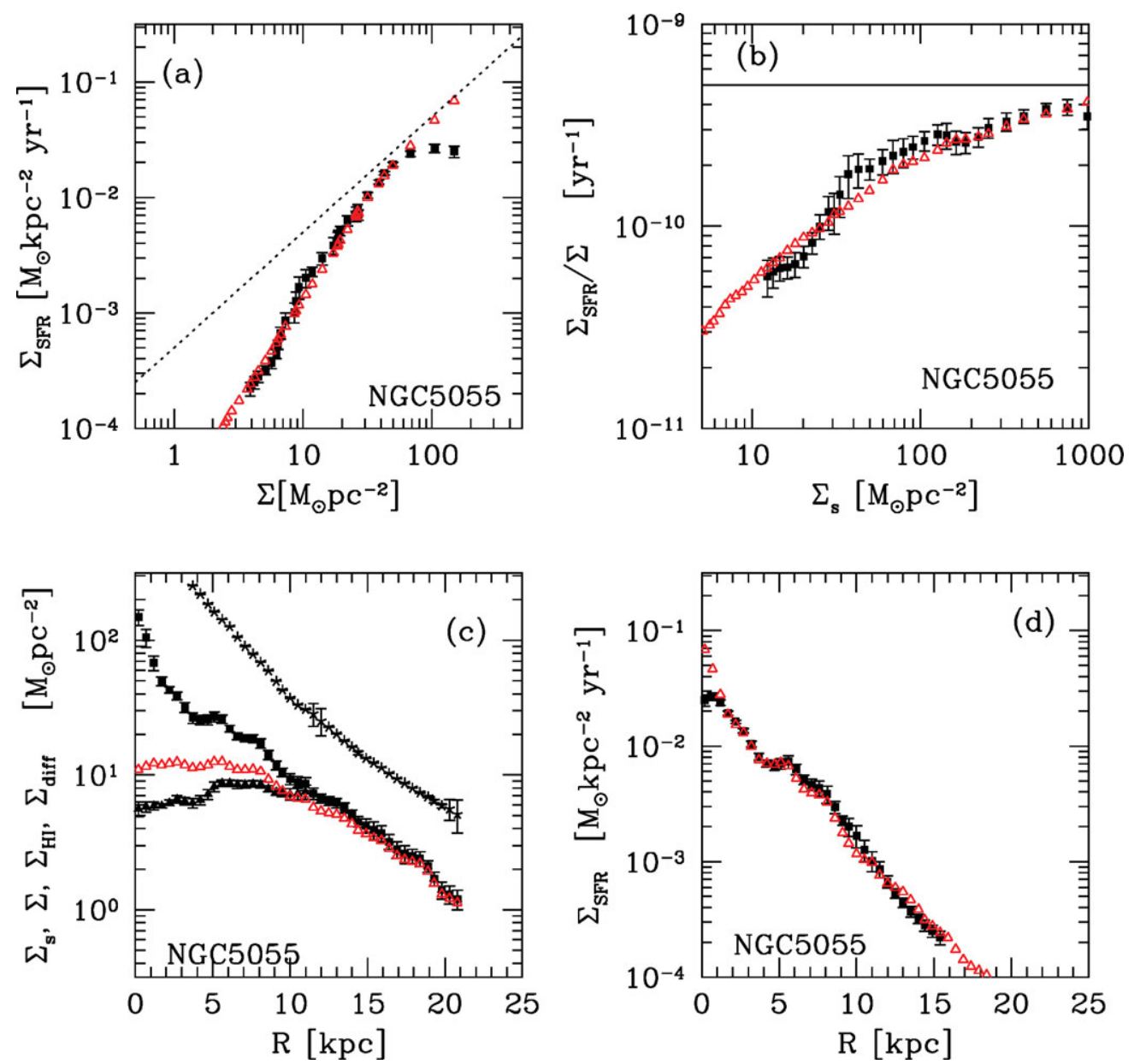

Figure 1. Comparison between annular averages of the data (squares) for NGC 5055 (Leroy et al. 2008), and the thermal/dynamical equilibrium model (triangles) developed in OML. Both the star formation rates as a function of radius in the galaxy (panel d), and star formation rates as a function of gas and stellar density (panels a and b) agree well with the model predictions.

$\rho_{\mathrm{Sd}} \propto \Sigma^{2 p}$ may hold such that $\Sigma_{\mathrm{SFR}} \propto \Sigma^{1+p}$ in outer disks, but this need not be the case in general - that is, integrated "Schmidt"-type relations need not hold.

In OML, the full solution for $\Sigma_{\mathrm{SFR}}$ is obtained as a function of $\Sigma, \rho_{\mathrm{sd}}$, and the parameters $\alpha \equiv P_{\text {tot }} / P_{\text {th }}$ and $\tilde{f}_{w} \equiv\left\langle v_{\text {th }}^{2}\right\rangle / c_{w}^{2}$, under the assumptions of thermal and dynamical equilibrium described above. It is also shown that the theoretical solution for $\Sigma_{\text {SFR }}$ agrees well overall with a sample of disk galaxies analyzed in Leroy et al. (2008), with especially close correspondence for the large flocculent galaxies NGC 7331 and NGC 5055. Figure 1 shows an example of the comparison between the model and data, for NGC 5055 .

Given the promising comparisons between the analytic theory and observations, it will be quite interesting to develop numerical simulations that fully test the assumptions and results of the thermal/dynamical equilibrium model. Encouragingly, the poster presented by C.-G. Kim at this meeting shows that initial numerical tests support the assumptions of thermal and dynamical equilibrium adopted in the analysis of OML. As discussed above, the OML theory contains parameters that must be set from either observations or detailed simulations. In the remainder of this contribution, we review what is known 
in this regard based on previous numerical work, and what measurements will be needed from future modeling efforts.

\section{Numerical evaluation of parameters}

From equations (2.1) and (2.3), the star formation rate in outer-disk regions is expected to vary as $\Sigma_{\mathrm{SFR}} \propto \Sigma \sqrt{2 G \rho_{\mathrm{Sd}}} \sigma_{z} / \alpha$, where $\alpha \equiv \sigma_{z}^{2} / v_{\text {th }}^{2}$ and $\sigma_{z}^{2}=v_{\mathrm{th}}^{2}+v_{\text {turb }}^{2}+(1 / 2) \Delta\left(v_{A}^{2}-\right.$ $\left.2 v_{A, z}^{2}\right)$, with $v_{\text {th }}^{2}, v_{\text {turb }}^{2}$, and $v_{A}^{2}$ the (mass-weighted) mean thermal, turbulent, and Alfvén speeds in the diffuse gas (we now omit angle brackets denoting averaging). The coefficient $\sigma_{z} / \alpha$ can also be written as $v_{\mathrm{th}}^{2} / \sigma_{z}=c_{w}^{2} \tilde{f}_{w} / \sigma_{z}$. Thus, the star formation rate is expected to depend on the total velocity dispersion $\sigma_{z}$ (or the ratio $\sigma_{z} / c_{w}$, where $c_{w}$ is fixed by atomic physics), and on the fraction of diffuse gas in the warm phase $\approx \tilde{f}_{w}$

The ratios $\sigma_{z} / c_{w}$ and $\tilde{f}_{w} \approx M_{\mathrm{diff}, \text { warm }} / M_{\mathrm{diff}, \text { total }}$ depend on the details of gas dynamics in the diffuse ISM. Important effects include warm and cold phase exchange via thermal instability; turbulence (with the associated shock heating and adiabatic temperature changes, as well as turbulent mixing); conversion of diffuse gas to GBCs via midplane settling, self-gravity, and turbulence-induced cloudlet collisions; return of GBC gas to the diffuse phase by photodissociation and by "mechanical" destruction processes (including expanding HII regions, winds, SNe, and radiation pressure). Turbulence in the diffuse gas can be driven by stellar energetic inputs as well as spiral shocks, the magnetorotational instability, large-scale gravitational instabilities in the disk, and cosmic infall.

Numerical studies to understand the various effects involved are very much a work in progress, but some consensus is already beginning to emerge on a number of points:

- For a medium with a bistable cooling curve, the midplane thermal pressure tends to evolve, by exchange of mass between cold and warm components of the diffuse phase, such that the mean value is comparable to, or slightly below, $P_{\text {two-phase }}$ (Piontek \& Ostriker 2005, 2007). Since out-of equilibrium effects depend on the heating time from shocks compared to the cooling time, the mean value of the thermal pressure, as well as the breadth of the pressure distribution, must in general be affected by the scale and the amplitude of turbulence (see Gazol et al. 2005, 2009; Audit \& Hennebelle 2005, 2010; Hennebelle \& Audit 2007; Joung \& Mac Low 2006; Joung et al. 2009). Realistic numerical evaluations of the mean thermal pressure (for a given radiative heating rate) therefore will require numerical simulations in which the vertical box size is comparable to the true scale height of the diffuse ISM, and in which the turbulent amplitude is $\sim 5-10 \mathrm{~km} \mathrm{~s}^{-1}$.

- Magnetic fields in differentially-rotating multiphase disks are amplified by the magnetorotational instability until the magnetic pressure becomes comparable to the thermal gas pressure, with $B_{z}^{2} \ll B^{2}$ (Piontek \& Ostriker 2005, 2007; Wang \& Abel 2009). Supernova-driven turbulence also contributes to amplifying the magnetic field (de Avillez \& Breitschwerdt 2005; Mac Low et al. 2005).

- The energy input from supernovae yield ISM velocity dispersions $\sim 5-10 \mathrm{~km} \mathrm{~s}^{-1}$ for models with a wide range of supernova driving rates and disk properties (e.g. Kim 2004; de Avillez \& Breitschwerdt 2005; Dib et al. 2006; Shetty \& Ostriker 2008; Agertz et al. 2009; Joung et al. 2009). These values are comparable to those observed in the HI gas. Simulations have also shown that the turbulent amplitudes decrease at smaller scales and for higher densities. With this range of turbulent velocity dispersions, the turbulent pressure in simulations of the diffuse ISM is comparable to the thermal pressure.

- The interaction between self-gravity and rotational shear also drives turbulence at significant levels $\left(\gtrsim 10 \mathrm{~km} \mathrm{~s}^{-1}\right.$ ) in galactic disks (Kim \& Ostriker 2001, 2007; Wada et al. 2002; Shetty \& Ostriker 2008; Tasker \& Tan 2009; Agertz et al. 2009; Aumer et al. 2010; Bournaud et al. 2010). However, the turbulent power is much larger at the large 
$(\sim \mathrm{kpc})$ scales that dominate the swing amplifier than at scales below the disk scale height, and in-plane velocities (which do not contribute to vertical support of the disk) are much larger than vertical velocities. Thus, turbulence driven by instabilities on large scales is likely of limited importance in regulating the effective midplane pressure (for a given local gas surface density $\Sigma$ ), and hence the star formation rate. (Gravitational instabilities would, however, enhance $\Sigma$ and thus $\Sigma_{\text {SFR }}$ locally.) Flapping associated with non-steady spiral shocks also drives turbulence in the diffuse ISM (Kim et al. 2006; Kim et al. 2010), but again, vertical motions are small compared to horizontal motions

Although numerical results have shown that the total turbulent velocity dispersion $\sigma_{z}$ is relatively insensitive to the disk properties and the supernova driving rate (consistent with observations), it is much less certain how the warm fraction, or $v_{\mathrm{th}}^{2}=\tilde{f}_{w} c_{w}^{2} \approx$ $c_{w}^{2} M_{\text {diff,warm }} / M_{\text {diff,total }}$, depends on disk conditions and/or the star formation rate. Assessing this dependence, including a full exploration of parameter space, is an important task for future numerical studies. The fraction of diffuse atomic gas in different phases is not well known empirically, either, although observations of C II with Herschel potentially afford a means to separate cold and warm components of the atomic medium (which both contribute to $21 \mathrm{~cm}$ emission).

Finally, it remains important to understand more fully how spiral structure develops, and in particular, whether it is possible to characterize in a simple way the fraction of gas in a given annulus that is found in "arm" vs. "interarm" conditions, and what the compression factor is for the gas surface density. Numerical simulations have begun to marry spiral structure with an increasingly realistic treatment of the ISM (including multiple phases, turbulence, and magnetic fields); much more, however, remains to be done on this front. It also remains to be determined how well models like that of OML apply locally for galaxies with strong spiral structure. More generally, it is important to assess which equilibria (thermal, dynamical, star formation) still apply locally even in galaxies with large-scale transient structure in the ISM (due to spiral arms, tidal interactions, mergers, cosmic inflows, etc.).

\section{Conclusion}

Gas is the raw material for star formation, but the detailed state of the ISM, which depends in turn on the internal galactic environment, determines the rate at which this material is processed to create new stars. Recent observations have begun to explore the correlation between gas content and star formation at increasingly high spatial resolution, revealing changes in star formation "laws" between inner and outer disks; other environmental dependences of star formation have also been explored, including intriguing correlations between molecular and stellar content of galactic disks.

Although the simplest recipes for star formation (such as a rate that depends inversely on the free-fall time at the mean ISM density) have difficulty matching the data, models that account for feedback and the multiphase character of the ISM are more successful. In particular, recent work suggests that the empirical correlation between molecular content and estimated midplane pressure can be understood as reflecting a state of simultaneous thermal and dynamical equilibrium in the diffuse ISM. The thermal/dynamical equilibrium model of OML develops the idea that UV from OB stars provides a feedback loop that regulates the star formation rate: the proportions of diffuse and self-gravitating gas adjust themselves so that the heating rate (proportional to the mass of self-gravitating gas) matches the cooling rate (proportional to the mass of diffuse gas and to the vertical gravitational field). The model formulated in OML is promising in terms of explaining star-forming behavior in observed systems. With numerical simulations, it will be 
possible to appraise - and potentially revise - the simplifying assumptions and parameterizations adopted by this equilibrium model. Time-dependent simulations will also lead to a much clearer understanding of how GBCs form and disperse, and how their properties and the formation/destruction timescales relate to galactic environment. This will aid in defining limits for applying equilibrium relations, while also pointing the way towards non-equilibrium theories of star formation.

Acknowledgements: This work was supported by grant AST-0908185 from the National Science Foundation, and by a fellowship from the John Simon Guggenheim Foundation. The author thanks the referee for a helpful report.

\section{References}

Agertz, O., Lake, G., Teyssier, R., Moore, B., Mayer, L., \& Romeo, A. B. 2009, MNRAS, 392, 294

Audit, E. \& Hennebelle, P. 2005, A\&\&A, 433, 1

-. 2010, A\&A, 511, A76+

Aumer, M., Burkert, A., Johansson, P. H., \& Genzel, R. 2010, ApJ, 719, 1230

Bournaud, F., Elmegreen, B. G., Teyssier, R., Block, D. L., \& Puerari, I. 2010, arXiv:1007.2566

Bigiel, F., Leroy, A., Walter, F., Brinks, E., de Blok, W. J. G., Madore, B., \& Thornley, M. D. 2008, AJ, 136, 2846

Blitz, L. \& Rosolowsky, E. 2006, ApJ, 650, 933

de Avillez, M. A. \& Breitschwerdt, D. 2005, A\& A, 436, 585

Dib, S., Bell, E., \& Burkert, A. 2006, ApJ, 638, 797

Field, G. B., Goldsmith, D. W., \& Habing, H. J. 1969, ApJL, 155, L149

Gazol, A., Luis, L., \& Kim, J. 2009, ApJ, 693, 656

Gazol, A., Vázquez-Semadeni, E., \& Kim, J. 2005, ApJ, 630, 911

Heiles, C. 2001, Tetons 4: Galactic Structure, Stars and the Interstellar Medium, 231, 294

Hennebelle, P. \& Audit, E. 2007, A\&A, 465, 431

Joung, M. K. R. \& Mac Low, M. 2006, ApJ, 653, 1266

Joung, M. R., Mac Low, M., \& Bryan, G. L. 2009, ApJ, 704, 137

Kim, C.-G., Kim, W.-T., \& Ostriker, E. C. 2006, ApJL, 649, L13

Kim, C., Kim, W., \& Ostriker, E. C. 2010, ArXiv e-prints

Kim, J. 2004, Journal of Korean Astronomical Society, 37, 237

Kim, W. \& Ostriker, E. C. 2007, ApJ, 660, 1232

Kim, W.-T. \& Ostriker, E. C. 2001, ApJ, 559, 70

Koyama, H. \& Ostriker, E. C. 2009a, ApJ, 693, 1316

-. 2009b, ApJ, 693, 1346

Leroy, A. K., Walter, F., Brinks, E., Bigiel, F., de Blok, W. J. G., Madore, B., \& Thornley, M. D. $2008, A J, 136,2782$

Mac Low, M., Balsara, D. S., Kim, J., \& de Avillez, M. A. 2005, ApJ, 626, 864

McKee, C. F., \& Ostriker, E. C. 2007, ARAA, 45, 565

Ostriker, E. C., McKee, C. F., \& Leroy, A. K. 2010, ApJ, 721, 975 (OML)

Piontek, R. A. \& Ostriker, E. C. 2005, ApJ, 629, 849

-. 2007, ApJ, 663, 183

Shetty, R. \& Ostriker, E. C. 2008, ApJ, 684, 978

Tasker, E. J. \& Tan, J. C. 2009, ApJ, 700, 358

Wada, K., Meurer, G., \& Norman, C. A. 2002, ApJ, 577, 197

Wang, P. \& Abel, T. 2009, ApJ, 696, 96

Wolfire, M. G., Hollenbach, D., McKee, C. F., Tielens, A. G. G. M., \& Bakes, E. L. O. 1995, ApJ, 443, 152

Wolfire, M. G., McKee, C. F., Hollenbach, D., \& Tielens, A. G. G. M. 2003, ApJ, 587, 278 